\title{
Induction of angiogenesis using VEGF releasing genipin-crosslinked electrospun gelatin mats
}

\author{
Costantino Del Gaudio ${ }^{\mathrm{a}, 1}$, Silvia Baiguera ${ }^{\mathrm{b}, 1}$, Margherita Boieri ${ }^{\mathrm{c}}$, Benedetta Mazzanti ${ }^{\mathrm{c}}$, \\ Domenico Ribatti $^{d, e}$, Alessandra Bianco ${ }^{a}$, Paolo Macchiarini ${ }^{\text {b,* }}$ \\ a Department of Industrial Engineering, University of Rome “Tor Vergata”, Intrauniversitary Consortium for Material Science and Technology (INSTM), \\ Research Unit Tor Vergata, Rome, Italy \\ ${ }^{\mathrm{b}}$ Advanced Center for Translational Regenerative Medicine (ACTREM), Karolinska Institutet, Stockholm, Sweden \\ ${ }^{\mathrm{c}}$ Department of Clinical and Experimental Medicine, University of Florence, Florence, Italy \\ ${ }^{\mathrm{d}}$ Department of Basic Biomedical Sciences, Neuroscience and Sensory Organs, Unit of Human Anatomy and Histology, University of Bari, Italy \\ e National Cancer Institute "Giovanni Paolo II", Bari, Italy
}

\section{A R T I C L E I N F O}

\section{Article history:}

Received 30 April 2013

Accepted 23 June 2013

Available online 15 July 2013

\section{Keywords:}

Angiogenesis

Cross-linking

Electrospinning

Gelatin mats

VEGF release

\begin{abstract}
A B S T R A C T
Rapid and controlled vascularization of engineered tissues remains one of the key limitations in tissue engineering applications. This study investigates the possible use of natural extracellular matrix-like scaffolds made of gelatin loaded with human vascular endothelial growth factor (VEGF), as a bioresorbable platform for long-term release and consequent angiogenic boosting. For this aim, gelatin was firstly electrospun and then cross-linked at two different concentrations $(0.1 \%$ and $0.5 \% \mathrm{w} / \mathrm{v})$ by using genipin, a low toxic agent, in order to fabricate a suitable substrate to be loaded with VEGF. Collected fibers were homogeneous and free of beads, the fibrous structure was retained after cross-linking. Mechanical properties were deeply affected by the chemical treatment showing a different behavior, depending on the testing conditions (i.e., dry or wet state). VEGF release was assessed by means of ELISA assay: a cumulative release of about $90 \%(0.1 \% \mathrm{w} / \mathrm{v})$ and $60 \%(0.5 \% \mathrm{w} / \mathrm{v})$ at 28 days was measured. Both VEGF loaded mats induced cell viability, endothelial differentiation and showed chemoattractive properties when tested on human mesenchymal stromal cells (hMSCs). In vitro and in vivo angiogenic assays demonstrated that the VEGF loaded mats induced an angiogenic potential in stimulating new vessel formation similar, if not superior, to fresh VEGF. VEGF retains bioactive and pro-angiogenic potential for up to 14 days. The results demonstrated that genipin cross-linked electrospun gelatin mats loaded with VEGF could be part of a useful strategy to stimulate and induce angiogenesis in tissue engineered applications.
\end{abstract}

(c) 2013 Elsevier Ltd. All rights reserved.

\section{Introduction}

The vascularization of a regenerating tissue, allowing cell and graft survival, has an essential role in the success of an implant: vessels, providing the construct not only with blood, but also with endothelial progenitor cells, play a key role for the survival of an engineered tissue. The identification of a strategy to obtain an effective vascularization of a construct is still one of the limiting steps in the field of tissue engineered devices, and to overcame this

\footnotetext{
* Corresponding author. Advanced Center for Translational Regenerative Medicine (ACTREM), Karolinska Institutet, Alfred Nobels Allé 8, Huddinge, SE-141 86 Stockholm, Sweden. Tel.: +46 760503213 (mobile); fax: +46 87747907.

E-mail address: paolo.macchiarini@ki.se (P. Macchiarini).

1 Contributed equally to the work.
}

drawback several approaches have been employed [1]. Structural parameters, such as scaffold pore size, have been varied to identify the most suitable condition for cellular adhesion and migration [2], cells have been included into the scaffold to initiate angiogenesis in vitro [3], or growth factors have been added into the scaffold to promote angiogenesis in vivo [4]. However, till now the most suitable strategy to develop sufficient vasculature has not been already identified. Recently, specific growth (such as Granulocyte colonystimulating factor) and boosting (such as Erythropoietin) factors have been used, both intraoperatively and post-operatively, in order to improve endothelial progenitor cell recruitment, to activate endogenous stem cells and to stimulate in situ vascularization and, as a consequence, tissue regeneration [5,6]. Despite the successful clinical results, new clinical strategies to drive and boost neoangiogenesis are still lacking and require further studies to provide 
suitable tissue regeneration. Moreover, it would be desirable to develop an approach providing controlled release of angiogenic factors to maintain high local therapeutic concentrations in tissue, while minimizing potential unwanted systemic effects.

A possible approach could be to administer growth factors by a slow-release system such as a bioresorbable scaffold. A scaffold fabricated by means of the electrospinning technique, that allows to collect polymeric mats made up of nano- or micrometric fibers with a consequent large surface-to-volume ratio that can enhance the incorporation of growth factors, can be then regarded as a valuable extracellular matrix (ECM)-like platform for the proposed aim. In addition, the use of natural polymers can further improve this strategy due to their intrinsic characteristics.

Gelatin, derived from collagen denaturation, is an attractive polymer for tissue engineering applications, being nonimmunogenic, bioresorbable, non-cytotoxic, and available at relatively low cost [7]. Gelatin sheets or sponges have been already evaluated, with promising results, as vectors to load growth factor [8-10]. However, being a water-soluble protein, gelatin needs to be properly treated in order to deal with a suitable scaffold. For this aim, cross-linking is an effective method that can ameliorate its starting characteristics, such as the mechanical performance and temporal stability. However, several concerns can be raised on the eventual cytotoxic effects of the compounds needed for this procedure. Glutaraldehyde, a common crosslinking agent that can significantly stabilize the treated polymer, results often associated with toxic effects. Genipin, a naturally crosslinking agent derived from the fruits Gardenia Jasminoides Ellis, resulting about 10,000 times less toxic than glutaraldehyde and providing a lower in vivo inflammatory response, can be regarded as a valuable alternative [11-13]. Recently, genipin has been used to improve the mechanical and pro-angiogenic properties of decellularized rat airway matrices, demonstrating that genipin crosslinked matrices were in vivo well accepted and did not induce any cytotoxic effects [14]. Cross-linking can also give a hydrogel-like behavior to the final substrate, allowing to load specific agents (i.e., drug and/or growth factors) within the structure, that can be subsequently released to stimulate and enhance peculiar biological processes. The resulting scaffolds, possessing a high water content, tunable mechanical properties and controllable degradation rates, could indeed represent a versatile strategy to deliver or present bioactive proteins.

Angiogenic processes are regulated by various growth factors and the vascular endothelial growth factor (VEGF) is the most potent one to promote the formation of new blood vessels. However, because of its short half-life (about $50 \mathrm{~min}$ ), high doses of VEGF are required, which resulted often associated to severe side effects (such as vasodilation and hypertension, inappropriate blood vessel growth, atherosclerotic plaque development and neovascularisation of tumors) $[15,16]$. On the other hand for the development of mature blood vessels, sustained local concentration of VEGF is necessary. Therefore, it results particularly important to develop an approach able to localize VEGF and control its release at the site of implantation.

The aim of this work was to evaluate the potential of genipin crosslinked electrospun gelatin mats as bioresorbable platforms for the long-term release of VEGF. In vitro and in vivo experiments were performed to assess the angiogenic potential of the released VEGF. The rationale of this approach was based on the fabrication of a substrate that could promote angiogenesis in a tissue engineered construct.

\section{Materials and methods}

\subsection{Materials}

Gelatin powder (type A, from porcine skin) was supplied by Sigma-Aldrich acetic acid was supplied by Carlo Erba Reagenti; genipin was supplied by Wako.
All materials and reagents were used as received.

\subsection{Scaffold fabrication}

\subsubsection{Electrospinning procedure}

Gelatin powder was dissolved in a mixture of acetic acid/deionized water (9:1), the concentration being $14 \% \mathrm{w} / \mathrm{v}$. Polymeric solution was poured into a glass syringe and then electrospun at room temperature through a blunt tip metallic needle $(22 \mathrm{G})$ at $12 \mathrm{kV}$ insured by a high voltage power supply (Spellman, USA) and at constant feed rate of $0.4 \mathrm{ml} / \mathrm{h}$ by means of a digital controlled infusion pump (KD Scientific, USA). The mat was collected onto a grounded aluminum target at $10 \mathrm{~cm}$ from the needle tip.

All samples were vacuum dried for $48 \mathrm{~h}$ and stored in a desiccator.

\subsubsection{Crosslinking procedure}

Genipin was dissolved in ethanol at two different concentrations, 0.1 and $0.5 \%$ $\mathrm{w} / \mathrm{v}$, respectively. Crosslinking was carried out by soaking the electrospun gelatin mats into the alcoholic solutions for 3 days at $37^{\circ} \mathrm{C}$. Subsequently, the resulting cross-linked scaffolds were rinsed in ethanol and dried at room temperature for $24 \mathrm{~h}$.

The investigated mats were labeled as follows: Gel (as-spun gelatin mat), Gel_Gen01 (cross-linked gelatin mat with $0.1 \%$ w/v genipin), and Gel_Gen05 (crosslinked gelatin mat with $0.5 \% \mathrm{w} / \mathrm{v}$ genipin).

\subsection{Mat characterization}

\subsubsection{Morphological characterization}

The microstructure of as-spun and cross-linked gelatin mats was investigated by means of scanning electronic microscopy (SEM; Leo-Supra 35). Samples were sputter coated with gold prior to examination. The average fiber diameter was determined from SEM micrographs by measuring about 50 fibers randomly selected (Image), $N I H)$. Two-dimensional void size evaluation, regarded as the average value of the equivalent diameters of the voids comprised among the polymeric fibers, was carried out as previously reported [17].

\subsubsection{Swelling}

Samples cut out from cross-linked mats were firstly weighed in air-died conditions and then immersed in $20 \mathrm{ml}$ of PBS at $37{ }^{\circ} \mathrm{C}$ and $95 \%$ relative humidity for different periods of time. Before weight measurements, wet samples were wiped with filter paper to remove the excess of solution. The swelling ratio was evaluated as:

$S W=\frac{W_{w}-W_{d}}{W_{d}}$

where $W_{w}$ and $W_{d}$ are the weights of wet and dried samples.

\subsubsection{Infrared analysis}

Fourier transform infra-red (FTIR) analysis of as-spun and cross-linked gelatin mats was performed by using a Perkin Elmer Spectrum 100. The spectra were collected in the range $4000-400 \mathrm{~cm}^{-1}$ at a resolution of $4 \mathrm{~cm}^{-1}$.

\subsubsection{Mechanical analysis}

Uniaxial tensile tests were carried out on rectangular specimens $\left(40 \times 5 \mathrm{~mm}^{2}\right)$ $(n=5)$ cut out from as-spun and cross-linked mats at ambient conditions (dry state) and after being soaked for $10 \mathrm{~min}$ in PBS prior to evaluation (wet state). Mechanical tests were performed at $5 \mathrm{~mm} / \mathrm{min}$ to rupture by means of a universal testing machine (UTM) equipped with a $100 \mathrm{~N}$ load cell (Lloyd LRX). The tensile modulus, the tensile strength (TS) and the strain at break were calculated from the stress-strain curves. Sample thickness was measured applying a pressure of $10 \mathrm{~g} / \mathrm{cm}^{2}$ by means of a custom-made set-up including a digital micrometer equipped with a $2 \mathrm{~N}$ load gauge (Imada, Inc., IL, USA).

\subsection{VEGF conditioning}

\subsubsection{VEGF loading}

Samples, as squared sheets $(5 \times 5 \mathrm{~mm})$, were previously sterilized by immersion in $100 \% \mathrm{v} / \mathrm{v}$ ethanol solution for $1 \mathrm{~h}$, dried in laminar hood at room temperature and incubated overnight with Hank's Balanced Salt Solution (HBSS) at $37^{\circ} \mathrm{C}$ in a humidified atmosphere with $5 \% \mathrm{CO}_{2}$. The solution of human VEGF(R\&D Systems, Minneapolis, MN) in HBSS + bovine serum albumin (BSA, $0.1 \%$ ) was then applied to the gelatin mats ( $50 \mathrm{ng} / \mathrm{mg}$ dry mat, $5 \mu \mathrm{l} / \mathrm{mg}$ of dry mat) and allowed to dry in laminar hood for $1 \mathrm{~h}$ at room temperature. Samples were then quickly washed with fresh HBSS.

\subsubsection{VEGF release}

The amount of VEGF released from samples was quantified using a human VEGF quantikine ELISA kit (R\&D Systems, Minneapolis, MN). After HBSS washing step, VEGF loaded samples were put into $200 \mu \mathrm{L}$ HBSS (+BSA 0.1\%) in 24-well plate at $37{ }^{\circ} \mathrm{C}$ in a humidified atmosphere of $5 \% \mathrm{CO}_{2}$. At specified time points the release 
medium was collected, replaced with fresh medium and the withdrawn medium stored at $-80{ }^{\circ} \mathrm{C}$. The release of bound VEGF was quantified up to 28 days.

\subsection{VEGF bioactivity}

The activity of VEGF was tested using human mesenchymal stromal cells (hMSCs). In order to assess the activity of VEGF loaded within gelatin cross-linked mats, four experimental conditions were evaluated: negative (cells incubated only with culture medium) and positive (fresh VEGF $(10 \mathrm{ng} / \mathrm{ml})$ added directly into the culture medium) controls and the two here considered testing conditions, i.e., Gel_Gen01 and Gel_Gen05 (VEGF loaded mats incubated by means of a cell strainer (8.0 $\mu \mathrm{m}$ PET membrane, BD Biosciences, Bedford, MA, USA) in order to be completely immersed in the medium but without touching and interfering with cell cultures).

In order to evaluate the activity of released VEGF, loaded mats were incubated in HBSS (+BSA $0.1 \%$ ) at $37{ }^{\circ} \mathrm{C}$ in a humidified atmosphere with $5 \% \mathrm{CO}_{2}$. After 7 or 14 days, HBSS was collected and stored at $-80^{\circ} \mathrm{C}$. The growth factor concentration was evaluated by the ELISA test. HBSS containing released VEGF was added directly into hMSC cultures, at the same concentration of fresh VEGF. Four experimental conditions were investigated: negative and positive controls (as above), cells incubated with culture medium added with HBSS containing released VEGF after 7 day incubation ( 7 day samples), and cells incubated with culture medium added with HBSS containing released VEGF after 14 day incubation (14 day samples).

\subsection{1. hMSC isolation}

hMSCs were obtained as previously described [18]. Briefly, cells were isolated from human bone marrow mononuclear cells (MNCs), which were seeded in Dulbecco's modified Eagle's medium with low glucose, (DMEM-LG; Gibco-Invitrogen, Milan, Italy), supplemented with $20 \%$ fetal bovine serum (FBS, Hyclone, SouthLogan, Utah) and $1 \%$ Penicillin-Streptomycin according to published methods [19,20]. The cells were incubated at $37^{\circ} \mathrm{C}$ in a humidified atmosphere with $5 \% \mathrm{CO}_{2}$. Cell characterization has been previously reported [18]. The third passage was used for the experiments.

\subsubsection{Cell viability}

hMSCs $\left(2.5 \times 10^{4}\right.$ cells $\left./ \mathrm{cm}^{2}\right)$ were seeded in standard medium(DMEM-LG, 20\% FBS, $1 \%$ Penicillin-Streptomycin). After $24 \mathrm{~h}$ of culture, the standard medium was replaced with culture medium (DMEM-LG, 1\% FBS, $1 \%$ Penicillin-Streptomycin) and the cultures incubated with VEGF loaded mats or with released VEGF for 24 h, 3 days and 7 days. After the incubation period, cells were detached and viability evaluation was performed using ADAM MC Automated Mammalian Cell Counter (Twin Helix, Mi, Italy).

\subsubsection{Cell migration assay}

To evaluate in vitro chemoattractant properties of VEGF loaded mats, the hMSC migration assay was performed using Transwell PET filters of $8.0 \mathrm{~mm}$ pore size $(B D$ Biosciences, Bedford, MA, USA). VEGF loaded mats were placed in the lower compartment of the chamber with $0.5 \mathrm{ml}$ DMEM-LG, 1\% FBS, 1\% Penicillin-Streptomycin. hMSCs were then seeded $\left(5 \times 10^{4}\right.$ cells $\left./ \mathrm{cm}^{2}\right)$ in the upper compartment in the same medium. The plates were incubated for $4 \mathrm{~h}$ at $37^{\circ} \mathrm{C}$. The filters were then removed, the upper surface was scraped, and cells that migrated towards the chemoattractant, remaining on the underside of the membrane, were stained with $4^{\prime}-6$ diamidino-2-phenylindole (DAPI). Five fields at $5 \times$ magnification were observed by fluorescence microscopy. Each sample was tested in triplicate.

\subsubsection{In vitro angiogenesis assay}

A morphogenic assay was performed by plating hMSCs on Matrigel (Becton Dickinson). Briefly, Matrigel was thawed on ice overnight, spread evenly over each well $(50 \mu \mathrm{L})$ of a 24 -well plate, and allowed to gel for $30 \mathrm{~min}$ at $37{ }^{\circ} \mathrm{C}$. hMSCs were seeded $\left(2.5 \times 10^{4}\right.$ cells $\left./ \mathrm{cm}^{2}\right)$ and cultured in culture medium (DMEM-LG, $1 \%$ FBS, $1 \%$ Penicillin-Streptomycin) containing or not containing fresh VEGF (10 ng/ml), VEGF loaded mats (by means of cell strainers), or released VEGF. After $24 \mathrm{~h}$, cultures were fixed with glutaraldehyde ( $2 \%$ ) and photographed ( 5 fields/well; the 4 quadrants and the central field) at a magnification of $\times 100$. Phase contrast images were recorded on a digital camera (Nikon, Tokio, Japan). Dimensional (percent area covered by hMSCs and total length of hMSCs network per field) and topological (number of meshes and branching points per field) parameters were estimated.

\subsubsection{In vivo pro-angiogenic properties}

The chicken embryo chorioallantoic membrane (CAM) assay was used as an in vivo model to evaluate the angiogenic properties of VEGF loaded mats. Fertilized White Leghorn chicken eggs ( $n=3$ for each condition) were incubated under constant humidity at $37^{\circ} \mathrm{C}$. On incubation day 3 , a square window was opened in the shell to detach the developing CAM after removal of $2-3 \mathrm{ml}$ of albumin. The window was sealed with a glass, and the eggs were returned to the incubator. At day 8 of incubation, $1 \mathrm{~mm}^{3}$ mat samples were placed on the CAM. $1 \mathrm{~mm}^{3}$ sterilized gelatin sponges (Gelfoam Upjohn, Kalamazoo Mich, USA) containing vehicle alone (PBS) were used as negative controls, while sponges containing $200 \mathrm{ng}$ of recombinant vascular endothelial growth factor A (VEGF-A, RED Systems, Abingdon, UK) were used as positive controls, as previously described $[21,22]$. All procedures were performed under sterile conditions. CAMs were examined daily until day 12 and photographed in ovo with a stereomicroscope equipped with a camera and image analyzer system (Olympus Italia, Milan, Italy). At day 12 the angiogenic response was evaluated as the number of vessels converging toward the implants and the sponges.

\subsubsection{Phenotypic analysis}

To evaluate in vitro differentiation potential of VEGF loaded mats or of released VEGF, treated hMSCs were analyzed for the expression of surface antigens using flow cytometry procedures [20]. hMSCs $\left(2.5 \times 10^{4}\right.$ cells $\left./ \mathrm{cm}^{2}\right)$ were seeded in standard medium (DMEM-LG, 20\% FBS, $1 \%$ Penicillin-Streptomycin). After $24 \mathrm{~h}$ of culture, the standard medium was replaced with culture medium (DMEM-LG, 1\% FBS, $1 \%$ Penicillin-Streptomycin) and the cultures incubated with VEGF loaded mats or with released VEGF for 7 days. After the incubation period, cells were detached and surface antigen expression evaluation performed. Washed cells were resuspended in flow cytometry buffer consisting of Cell WASH $(0.1 \%$ sodium azide in PBS; $B D$ Pharmigen, San Jose, CA, USA ) with $2 \%$ FBS. Aliquots $\left(1.5 \times 10^{5}\right.$ cells $\left./ 100 \mu \mathrm{L}\right)$ were incubated with the following conjugated MoAb: CD44-FITC, CD90-PE, HLA-ABCFITC (all from BD Pharmingen) and KDR-PE (RED System, Minneapolis, MN, USA). Non-specific fluorescence and morphologic parameters of the cells were determined by incubation of the same cell aliquot with isotype-matched mouse MoAb (BD Pharmingen). All incubations were done for $15 \mathrm{~min}$ and, after incubation, cells were washed and resuspended in $100 \mu \mathrm{L}$ of Cell WASH; 7-AAD (7-Aminoactinomycin-D) was added in order to exclude dead cells from the analysis. Flow cytometric acquisition was performed by collecting $10^{4}$ events on a FACSCanto (Becton Dickinson, Milan, Italy) and data were analyzed on DIVA software (Becton Dickinson).

\subsection{Statistics}

Results are expressed as mean \pm standard deviation. Assays was performed in triplicate. Data analysis was performed with nonparametric tests (SPSS 19.0, SPSS Inc., USA). Statistical analysis to assess differences between groups was performed in two steps. First, data were compared by using the Kruskal-Wallis nonparametric test. If significant differences were found, groups were compared individually by using the Mann-Whitney $U$ test. $p$ values less than 0.05 were considered significant.

\section{Results}

\subsection{Morphological characterization}

Electrospun gelatin mats were characterized by randomly arranged homogeneous fibers free of beads (Fig. 1). The average fiber diameter was $0.22 \pm 0.04 \mu \mathrm{m}$, while the average void size was $0.74 \pm 0.30 \mu \mathrm{m}$. Cross-linked mats were still characterized by a fibrous structure, even if fiber diameters were larger than those of the as-spun case (i.e., $0.60 \pm 0.16 \mu \mathrm{m}$ and $0.55 \pm 0.14 \mu \mathrm{m}$ for Gel_Gen01 and Gel_Gen05, respectively). Moreover, fused regions were observed at the overlapping fiber sites.
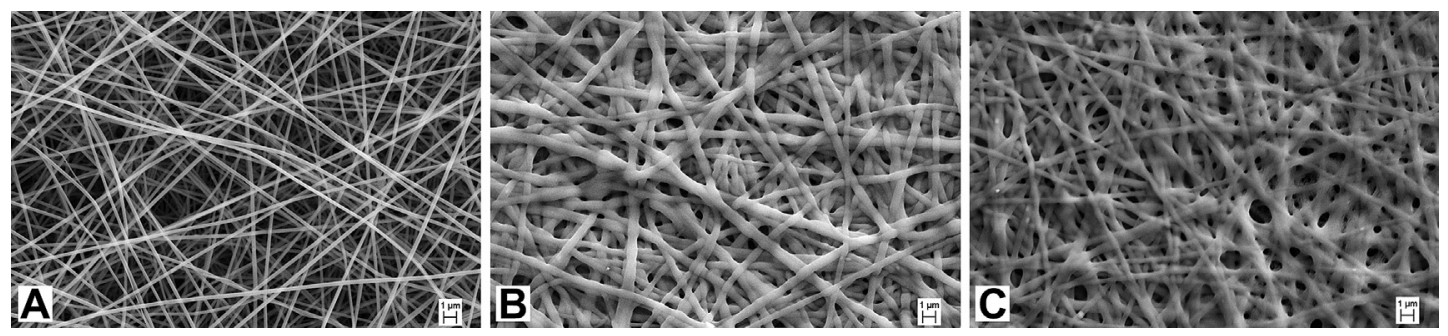

Fig. 1. SEM micrographs of as-spun gelatin (A), cross-linked gelatin mat with $0.1 \% \mathrm{w} / \mathrm{v}$ genipin (B), and cross-linked gelatin mat with $0.5 \% \mathrm{w} / \mathrm{v}$ genipin (C). 


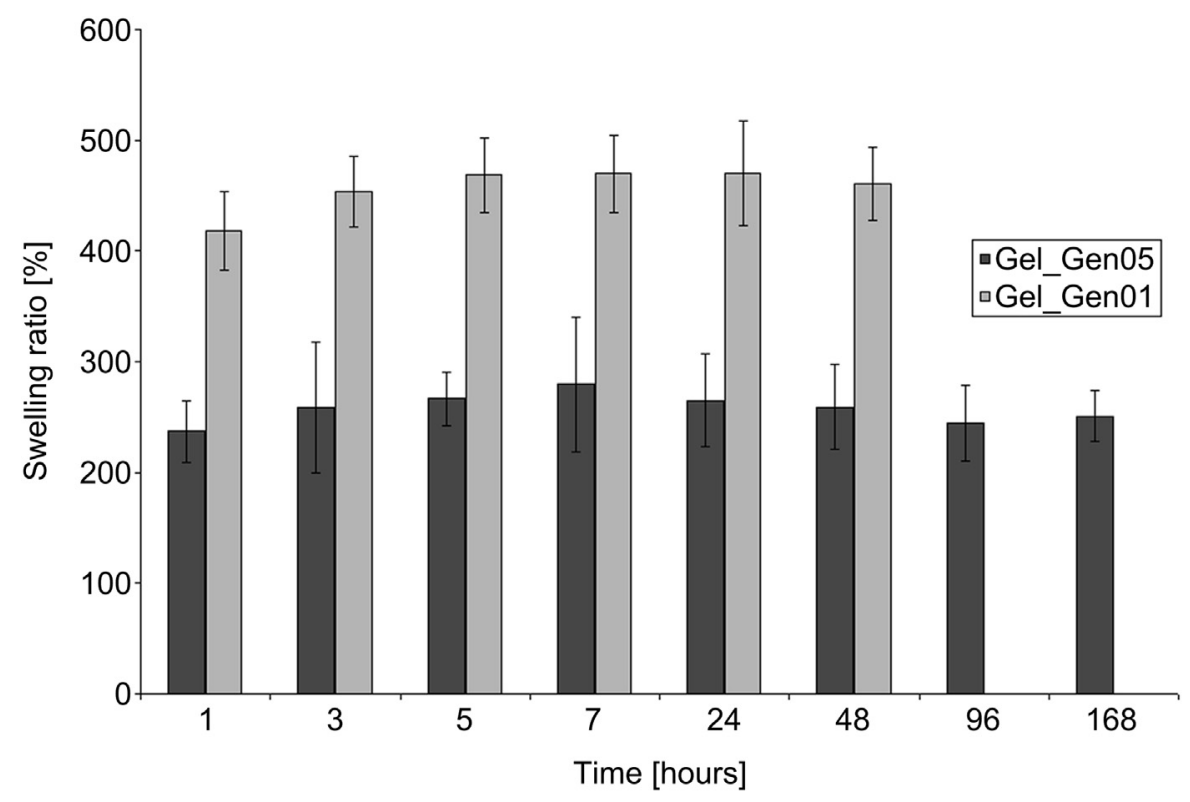

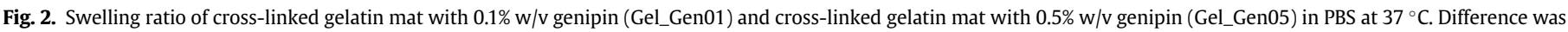
statistically significant $(p<0.05)$ for each considered time-point.

\subsection{Swelling}

Swelling assessment is reported in Fig. 2. Both cross-linked mats were characterized by a burst mass increase in the very first period followed by a constant behavior. An inverse relationship between swelling properties and genipin content was also assessed, Gel_Gen01 being characterized by a higher swelling ratio. In addition, this case showed a limited temporal stability to saline solution, starting to degrade from the 4 th day.

\subsection{Infrared analysis}

Infrared spectra of the investigated samples are shown in Fig. 3. Similar spectra were assessed for all the cases here investigated. Amide A peak (N-H stretching mode) was detected at $3300 \mathrm{~cm}^{-1}$ for Gel, while for Gel_Gen01 and Gel_Gen05 was little shifted at $3320 \mathrm{~cm}^{-1}$. Amide I (predominantly $\mathrm{C}=\mathrm{O}$ stretching mode, with contributions from in-phase bending of the $\mathrm{N}-\mathrm{H}$ bond and stretching of the $\mathrm{C}-\mathrm{N}$ bond $)$ and III $(\mathrm{C}-\mathrm{N}$ stretching mode) were

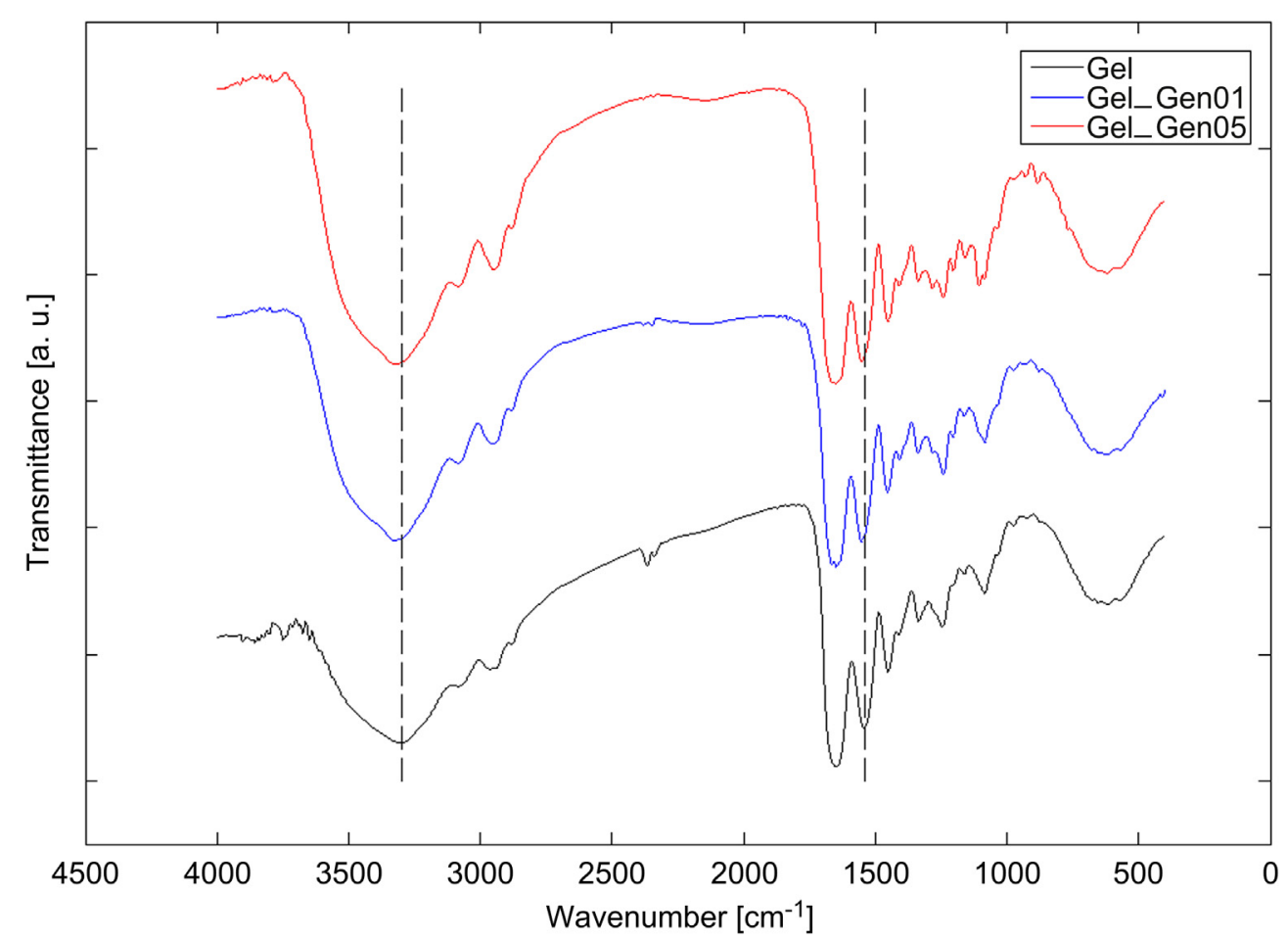

Fig. 3. FTIR spectra of as-spun gelatin (Gel), cross-linked gelatin mat with $0.1 \%$ w/v genipin (Gel_Gen01), and cross-linked gelatin mat with $0.5 \%$ w/v genipin (Gel_Gen05). 
Table 1

Mechanical properties of electrospun gelatin scaffolds as dependent on genipin cross-linking concentrations either in dry or wet state.

\begin{tabular}{lccc}
\hline & $\begin{array}{l}\text { Tensile modulus } \\
(\mathrm{MPa})\end{array}$ & $\begin{array}{l}\text { Stress at break } \\
(\mathrm{MPa})\end{array}$ & $\begin{array}{l}\text { Strain at } \\
\text { break }(\%)\end{array}$ \\
\hline Gel & $29.30 \pm 4.50$ & $1.00 \pm 0.05$ & $7.10 \pm 1.00$ \\
Gel_Gen01 dry state & $496.41 \pm 89.60^{*}$ & $17.22 \pm 6.26^{*}$ & $3.88 \pm 0.88^{*}$ \\
Gel_Gen05 dry state & $845.21 \pm 100.50^{*, 5}$ & $32.05 \pm 10.45^{*}$ & $4.40 \pm 1.03^{*}$ \\
Gel_Gen01 wet state & $0.57 \pm 0.13^{*}$ & $0.24 \pm 0.07^{*}$ & $34.61 \pm 19.24^{*}$ \\
Gel_Gen05 wet state & $1.10 \pm 0.20^{*}, \circ$ & $1.22 \pm 0.68^{\circ}$ & $54.42 \pm 15.03^{*}$ \\
\hline
\end{tabular}

${ }^{*} p<0.05$ with respect to Gel.

${ }^{\S} p<0.05$ with respect to Gel_Gen01 dry state.

$p<0.05$ with respect to Gel_Gen01 wet state.

located at $1650 \mathrm{~cm}^{-1}$ and $1240 \mathrm{~cm}^{-1}$, respectively, with no significant shifts for the three samples. Finally, a little shift was also detected for amide II ( $\mathrm{N}-\mathrm{H}$ bending mode), from $1540 \mathrm{~cm}^{-1}$ for the as-spun sample to $1550 \mathrm{~cm}^{-1}$ for the cross-linked ones [23,24].

\subsection{Mechanical characterization}

Electrospun gelatin specimens were mechanically tested before and after the cross-linking procedure. The mechanical behavior was dramatically affected by the experimental conditions, i.e., as-spun and cross-linked samples either in dry and swollen state. In the first case, tensile modulus and stress at break significantly increased with genipin concentration compared to as-spun gelatin, while an opposite trend was verified in the latter case, also showing a relevant increase of strain at break values. Table 1 summarizes the tensile modulus, stress and strain at break acquired from the stress-strain curves for each condition.

\subsection{Controlled release of VEGF}

The cumulative release trend of VEGF by both cross-linked mats resulted similar: a burst release within the first day of incubation followed by a prolonged sustained release. After 1 month the Gel_Gen01 and Gel_Gen05 released about 90\% and 60\% of the initial VEGF content, respectively (Fig. 4).

\subsection{Effect of loaded and released VEGF on hMSC viability}

As shown in Fig. 5, all the evaluated conditions revealed an increased viability, significantly different $(p<0.05)$ at 7 days, compared to negative control cultures. Moreover, an increased cell viability, significantly ( $p<0.05$ ) different at 3 days, was induced by both types of gelatin mats with respect to that induced by positive cultures, suggesting that the VEGF loaded on mats has a higher cellular effect to fresh VEGF. A similar effect was obtained when evaluating released VEGF, confirming that the factor retained its potential effect also after being released from gelatin cross-linked mats after 7 and 14 days of incubation (Fig. 6).

\subsection{Effect of loaded VEGF on hMSC migration and capillary tube formation}

Both types of VEGF loaded mats resulted to have in vitro chemoattractive properties (Fig. 7). In particular the migration induced by the Gel_Gen01 resulted to be significantly $(p<0.05)$ higher compared both to negative and positive controls.

The pro-angiogenic activity of loaded VEGF mats was evaluated by the Matrigel capillary-like structure formation assay (Fig. 8): negative control cultures did not formed capillary-like tubes (Fig. 8A), while treatment with fresh VEGF (Fig. 8B) or with VEGF loaded mats (Fig. $8 \mathrm{C}-0,1 \%$, Fig. 8D $-0,5 \%$ ) promoted hMSC spreading and alignment to form branching anastomotic tubes, which gave rise within $18 \mathrm{~h}$ to a mesh of capillary-like structures. Image analysis confirmed these observations, showing that all treatments increased dimensional (percent area covered by hMSCs and total length per field (length)) and topological parameters (number of mesh per field (mesh number) branching points per

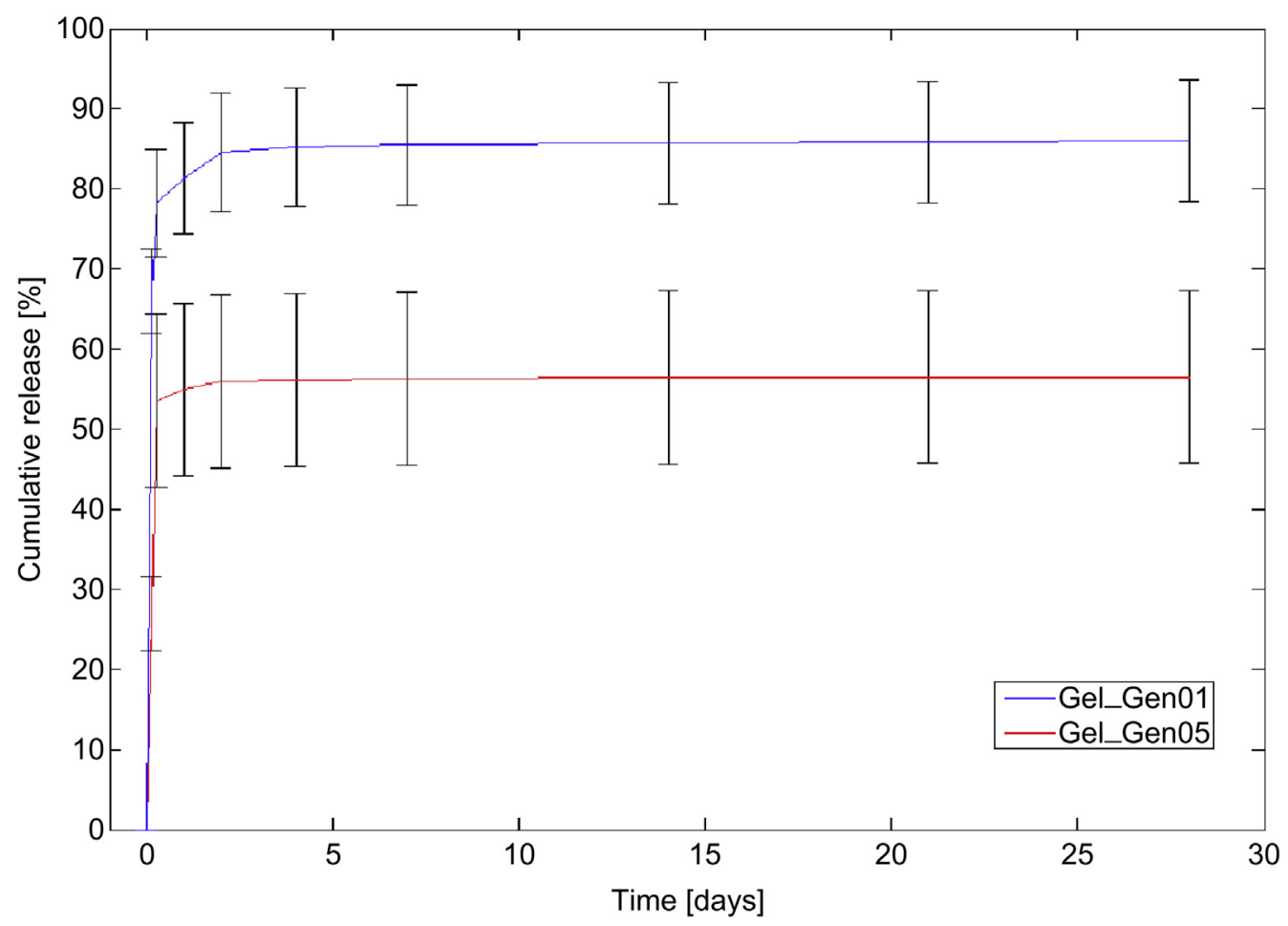

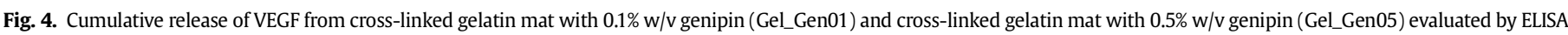
assay. 


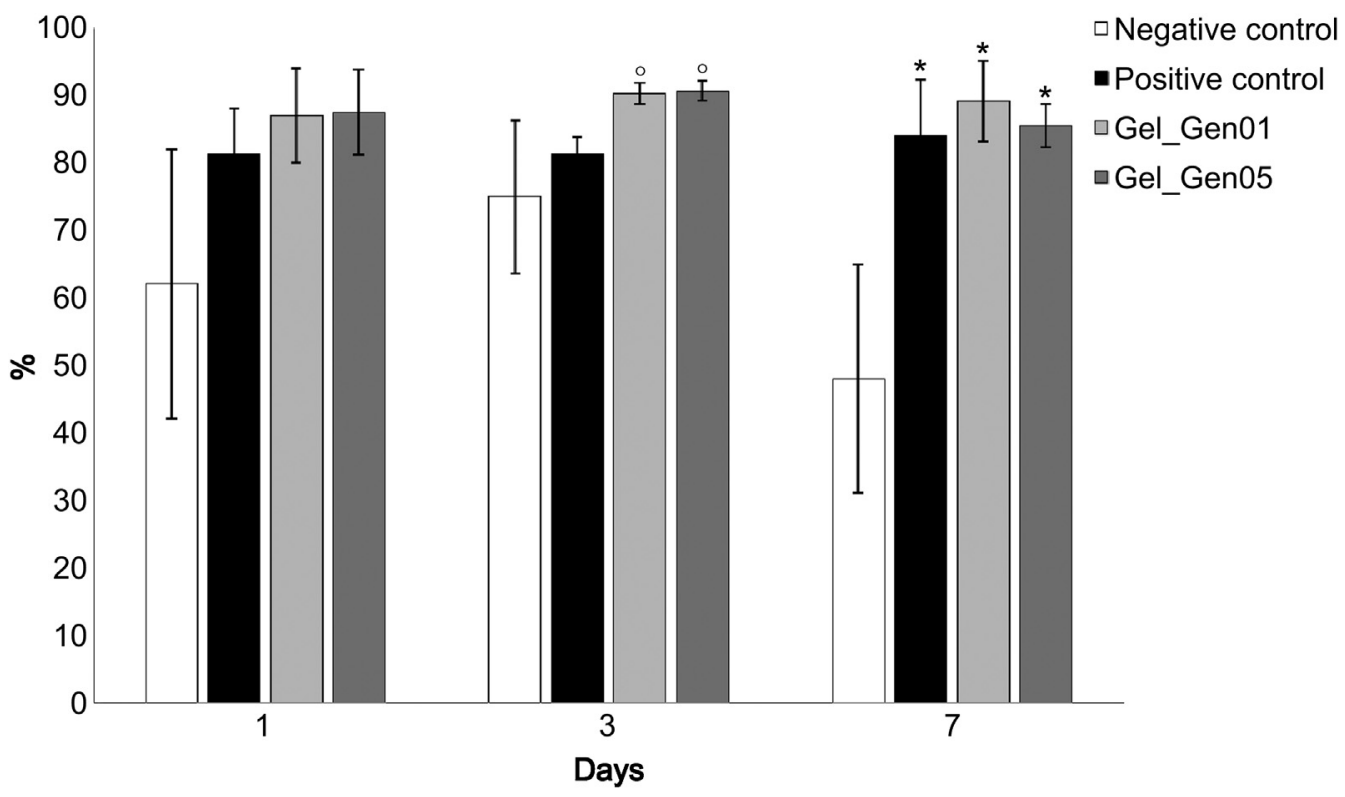

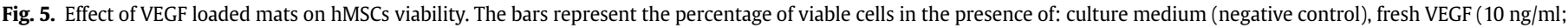

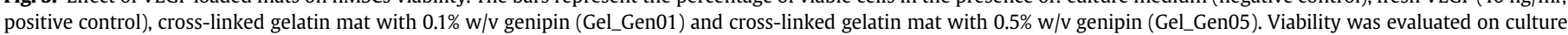
day 1 , day 3 and day $7 .{ }^{*} p<0.05$ versus negative control, ${ }^{\circ} p<0.05$ versus positive control.

field (branching)) of hMSCs capillary-like network (Fig. 8E). In particular, Gel_Gen0.1 induced an angiogenic effect significantly $(p<0.05)$ higher respect to that induced by fresh VEGF.

Similar results were obtained when evaluating the proangiogenic effect of released VEGF (Fig. 9): hMSCs incubated with released VEGF (Fig. 9C -7 days, Fig. 9D -14 days) gave rise to a meshwork of capillary-like structures, which resulted more organized with respect to negative and positive cultures (Fig. 9A,B), as image analysis confirmed (Fig. 9E). These results confirmed that VEGF, both loaded and released from gelatin cross-linked mats, retained its angiogenic potential.

\subsection{In vivo assay}

The in vivo assessment of the potency of the VEGF-loaded mats was performed by chick chorioallantoic membrane (CAM) assay. Macroscopic observations of CAM treated with VEGF loaded mats showed that all samples were surrounded by allantoic vessels that developed radially towards the implant in a spoke-wheel pattern. New vessels developed towards the VEGF loaded mats and were sometimes arranged in loops around the samples, positively affecting the growth and the organization of the network of CAM vessels (Fig. 10A). The angiogenic effect on direct blood vessel growth was

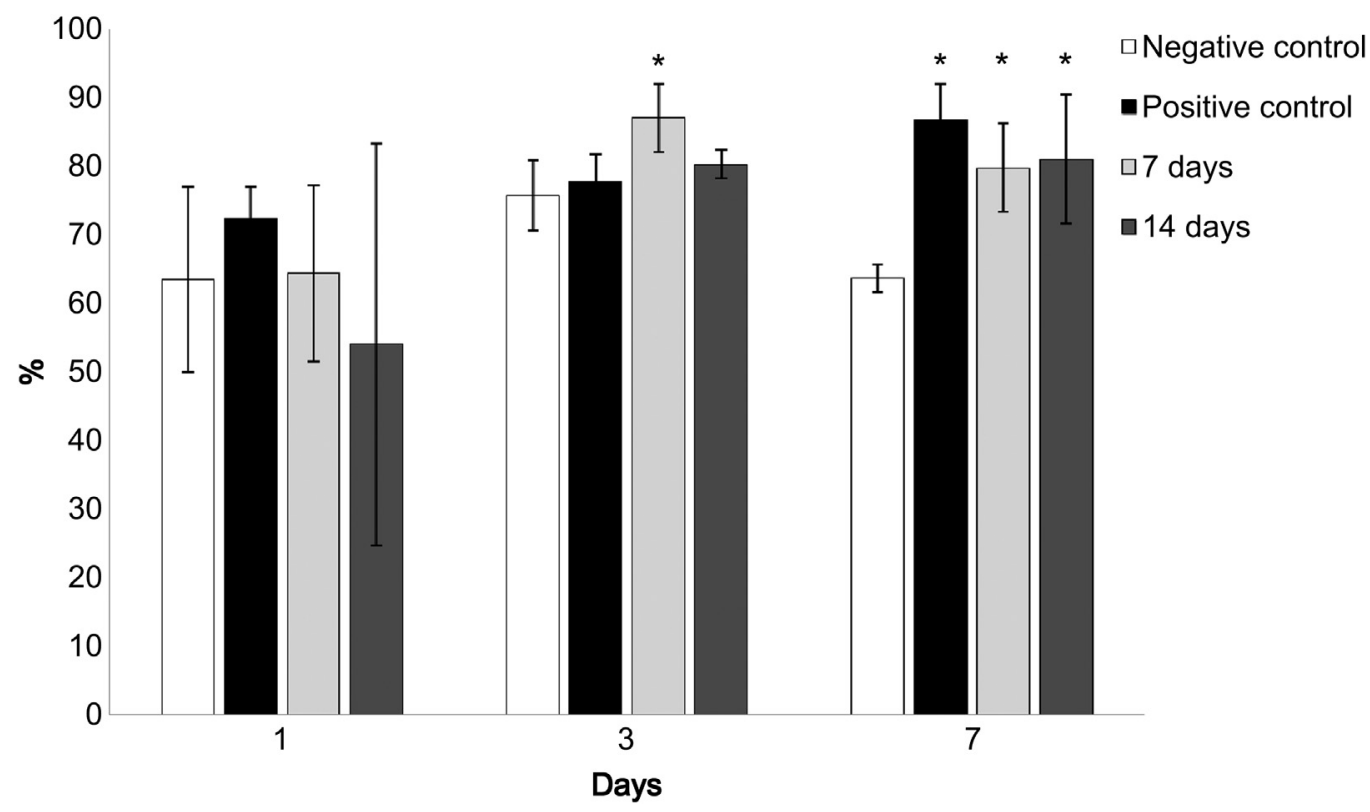

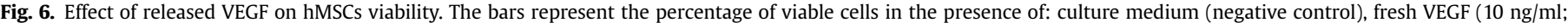

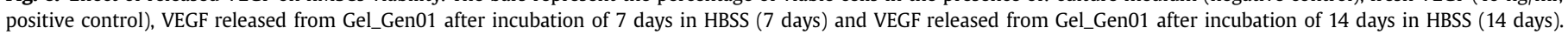
Viability was evaluated on culture day 1 , day 3 and day $7 .{ }^{*} p<0.05$ versus negative control. 

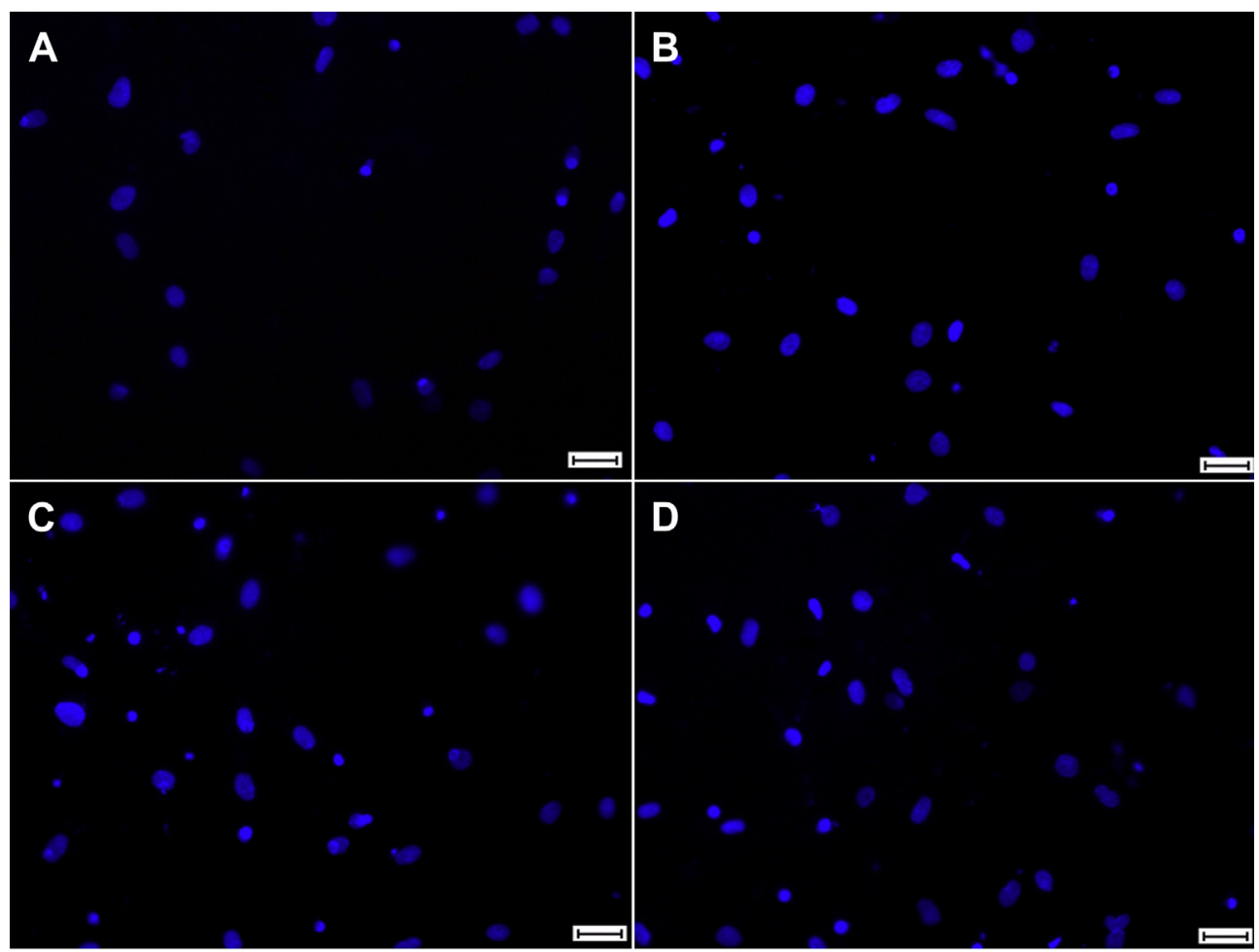

E

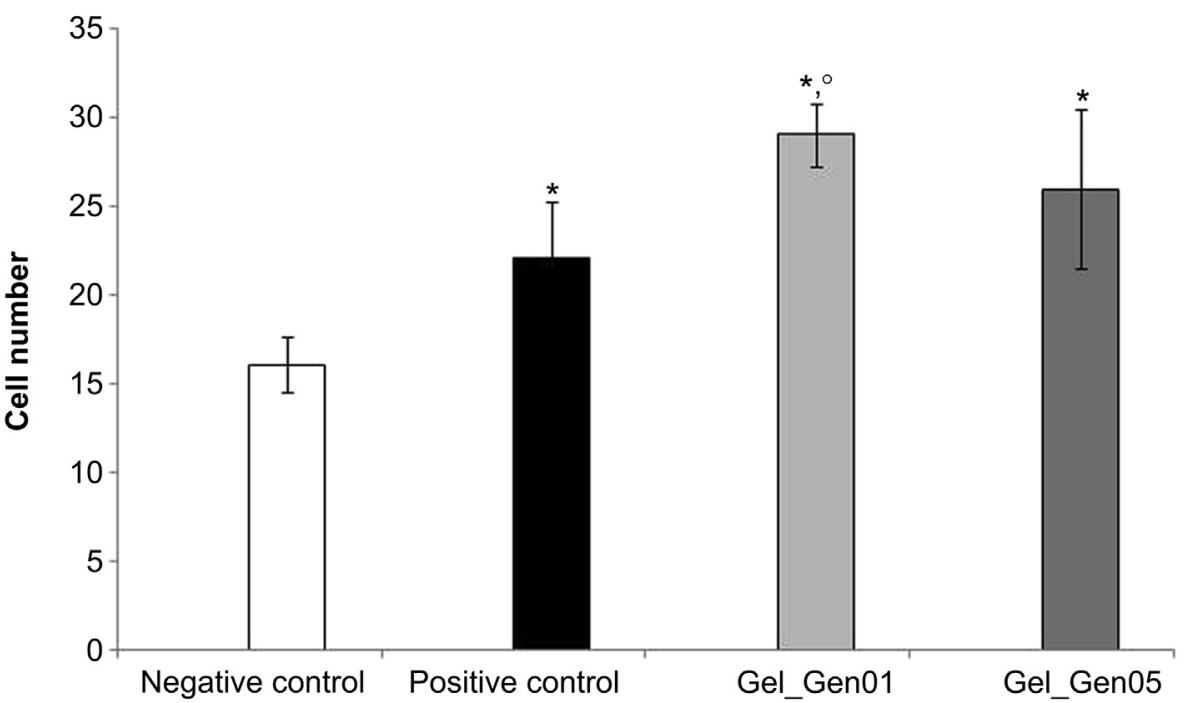

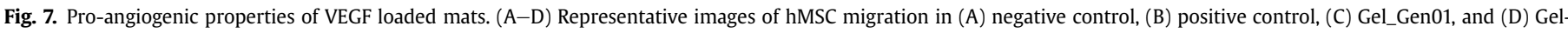

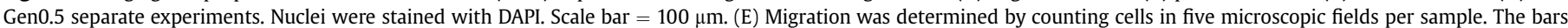

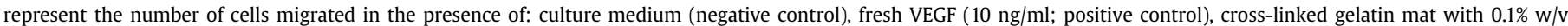
genipin (Gel_Gen01) and cross-linked gelatin mat with $0.5 \% \mathrm{w} / \mathrm{v}$ genipin (Gel_Gen05). ${ }^{*} p<0.05$ versus negative control, ${ }^{\circ} p<0.05$ versus positive control.

quantified as the total number of blood converging vessels (Fig. 10B). VEGF loaded mats induced a very similar effect, which resulted to be significantly $(p<0.05)$ higher compared to that induced by PBS (used as negative control), and comparable to the one induced by VEGF (used as positive control), suggesting that VEGF loaded on mats has in vivo angiogenic properties similar to fresh VEGF.

\subsection{Phenotypic analysis}

Flow cytometric analysis was used to evaluate antigen expression of hMSCs treated with VEGF loaded mats or VEGF released from mats. The results showed that the expression of hMSC surface antigens was not altered after a 7-day incubation with loaded or released VEGF, yielding hMSCs uniformly positive for CD44, CD90 and HLA-ABC (Table 2). Moreover, the expression of KDR, the main mediator of VEGF-induced endothelial proliferation, survival, migration, tubular morphogenesis and sprouting, resulted significantly $(p<0.05)$ higher in all VEGF treated cells respect both to negative and positive controls, suggesting a differentiation potential of VEGF towards endothelial pathway (Fig. 11).

\section{Discussion}

The use of selected angiogenic growth factors (such as VEGF) may be a useful approach for enhancing angiogenesis. However, their applicability results often limited by the factor short half-life 

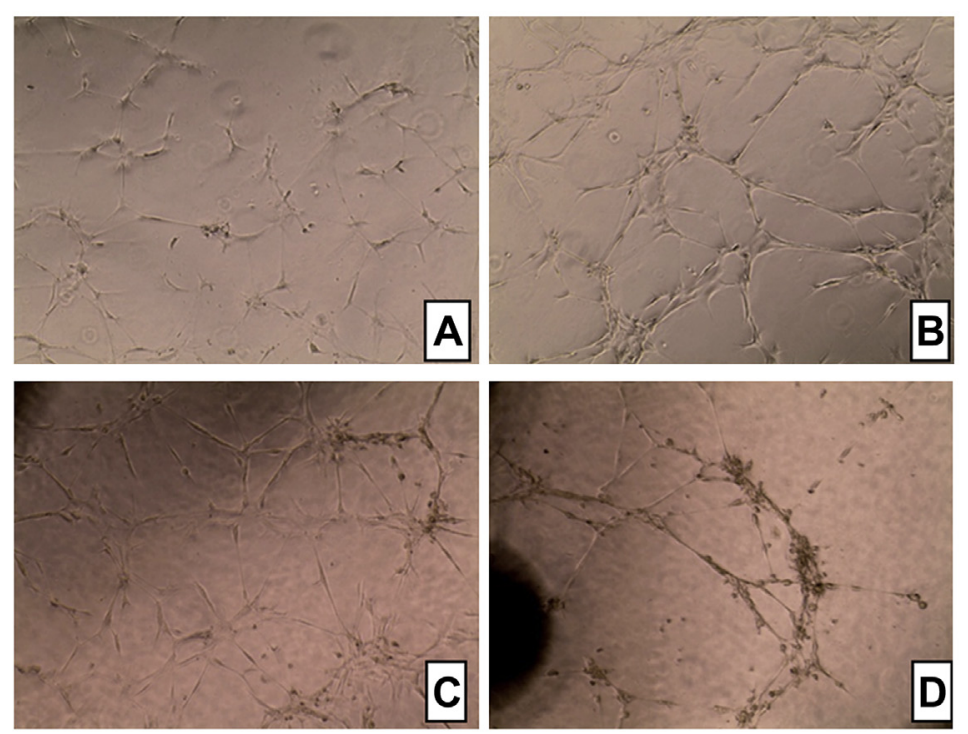

Branching points

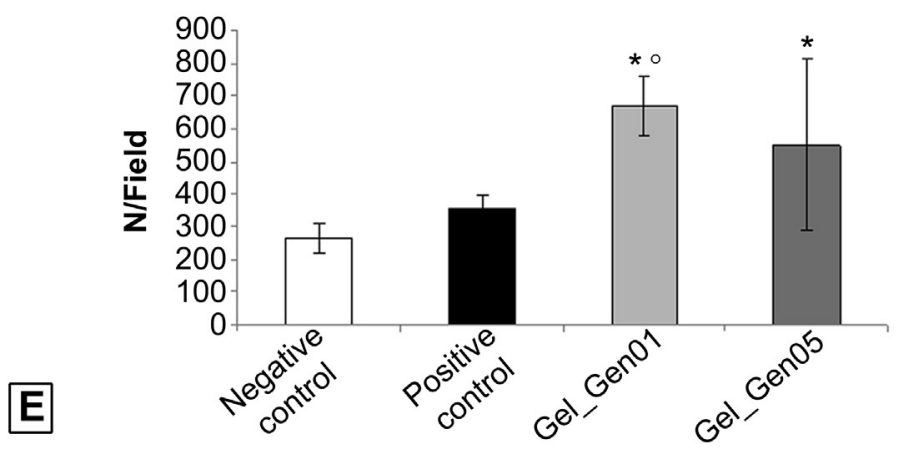

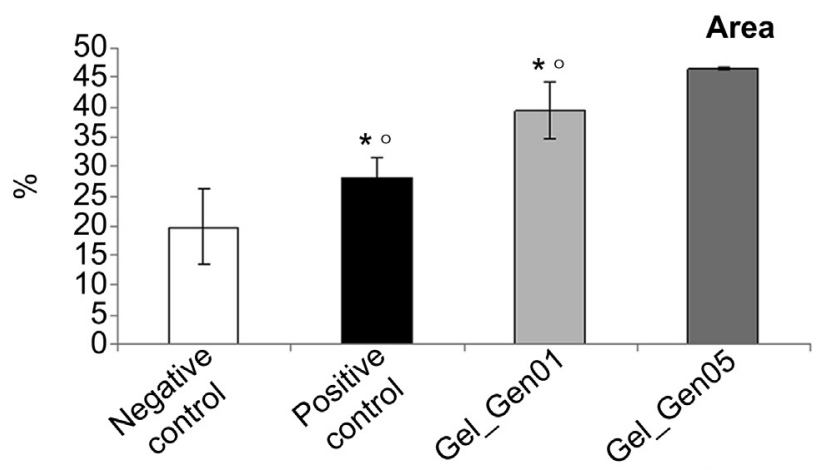
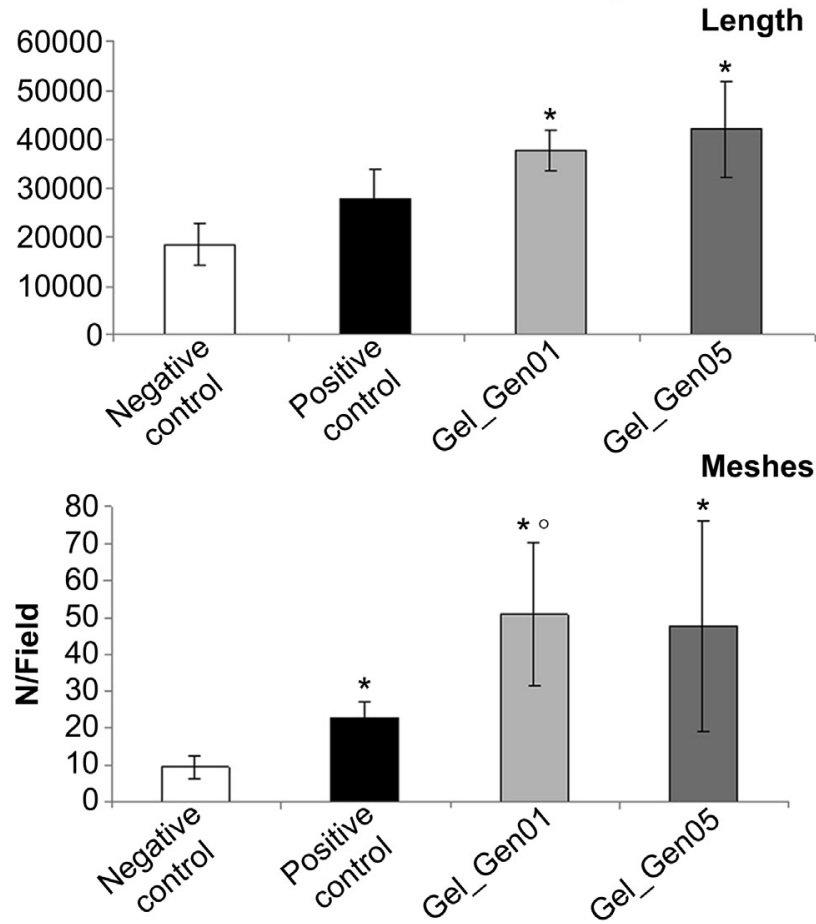

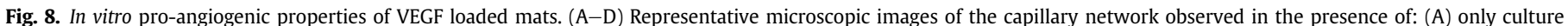

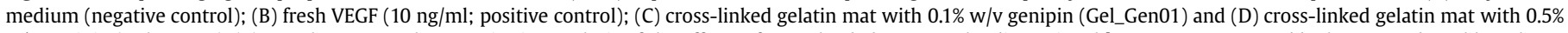

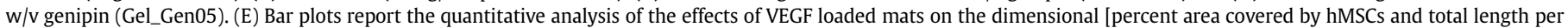

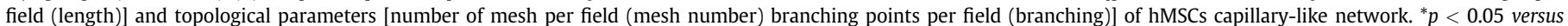
negative control, ${ }^{\circ} \mathrm{p}<0.05$ versus positive control.

and the rapid clearance from the implant site. The achievement of a matrix combining high loadability with controlled release of angiogenic factor still represents a major challenge in the field of tissue engineering.

Natural polymers represent a valuable alternative for biomedical applications, due to their origin and the related intrinsic characteristics that can elicit a positive host response. However, it is well stated that several drawbacks can be listed as well, generally referred to the mechanical properties and degradation profile that counterbalance the advantages of this kind of biomaterials. In order to address this issue, cross-linking can be a suitable procedure for the fabrication of natural scaffolds characterized either by specific biological cues and sufficient structural stability [25]. For this aim, genipin cross-linked electrospun gelatin mats were here fabricated and investigated as a potential VEGF-release platform to induce angiogenesis. The collected microarchitecture was composed of randomly uniform fibers that was retained after cross-linking, even if a modification in terms of fiber dimension was clearly observed. This result was already reported when similar cross-linking conditions were considered [26,27]. Interestingly, a distinct fiber morphology was obtained after cross-linking gelatin with glutaraldehyde vapor [28]. However, this approach might represent a limitation due to the toxic effects of this agent, especially if the main goal, as stated by the Authors themselves, was the fabrication of electrospun gelatin mats by using non toxic solvents (i.e., a mixture of ethanol and phosphate buffer saline solution). An improvement can be obtained by dissolving gelatin in distilled water, electrospinning the resulting solution in an ad hoc set-up, and then cross-linking the collected mats by immersion in a solution of 1-ethyl-3-(dimethyl-aminopropyl) carbodiimide hydrochloride and N-hydroxyl succinimide, even if a modification of the microstructure was observed after the chemical treatment [29].

The effectiveness of cross-linking was clearly assessed by mechanical tests. Both genipin concentrations improved the response of gelatin mats in the dry state, while an opposite behavior was evaluated in the wet state, compared to the as-spun case. This difference should be critically considered since the improvement of the mechanical performance, as clearly demonstrated by a number of previous studies by using different cross-linking agents [26,30], is related to the testing conditions that are not representative of the 

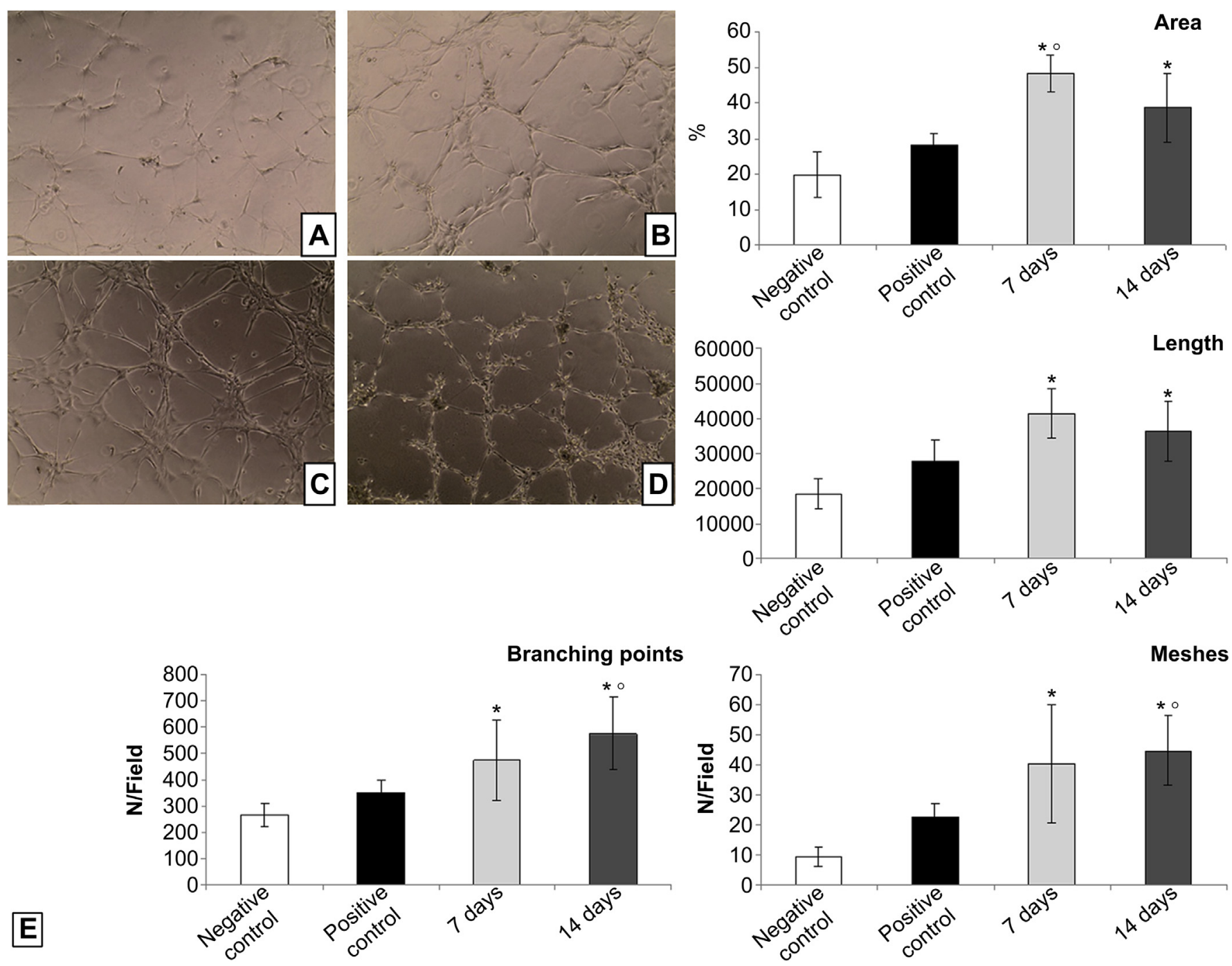

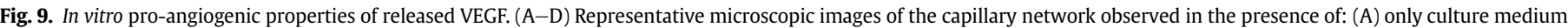

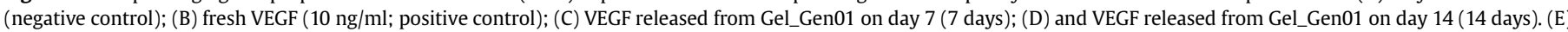

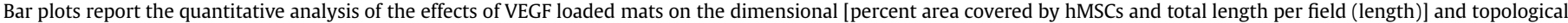

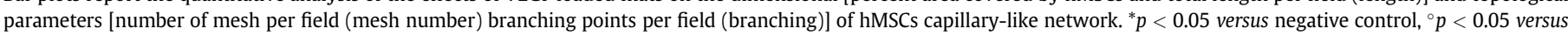
positive control.

biological environment, i.e. either in vitro or in vivo conditions cannot be referred to a "dry state". A similar approach, to simulate a "biologic" environment, was previously considered to mechanically test electrospun chitosan scaffolds cross-linked with genipin immediately upon removal from PBS [31]. In addition, the computed parameters might be more representative of a physiological environment suitable for endothelial cell differentiation/ colonization and angiogenesis promotion [32-34]. To further enhance the final characteristics of a tissue engineered scaffold that might have an effective biomedical potential, numerous strategies have been developed to localize VEGF within its microstructure. Encapsulation, entrapment, or covalent binding are the more robust approaches in achieving predictive drug release, however they involve multiple steps during scaffold fabrication that may compromise protein's bioactivity. On the other hand, adsorption of growth factor on prefabricated scaffold is a convenient, straightforward alternative that circumvents exposure to harsher conditions during scaffold preparation [35]. It has been demonstrated that the physical adsorption of VEGF to various surfaces improved angiogenesis in vivo [35-39]. In this work cross-linked gelatin mats loaded with VEGF by adsorption were presented, which resulted able to promote angiogenic processes through its sustained releases (i.e., 28 days).

Before any experiment, the mats were prewashed with HBSS to eliminate the sudden burst release phase due to not linked factor. Results showed that the adsorbed VEGF is released from both types of scaffolds in two phases: a burst response within the first day, followed by a nearly constant release until the end of the experimental period. A higher cumulative release was measured for Gel_Gen01 that can be associated to the higher swelling ratio and to the related medium diffusion. The cumulative release rate of VEGF resulted to be about 30\% lower for Gel_Gen05 respect to Gel_Gen01, suggesting that the magnitude of the VEGF release rate could be modulated by varying the cross-linking concentration. Several scaffold fabrication strategies could be proposed in order to modulate the subsequent release, based, e.g., on the development of suitable loading conditions of the growth factor that, however, should not be exposed to the harsh conditions of a typical production process. Differently from the swelling test, the VEGF release assay was performed using HBSS instead of PBS, being HBSS the 
A

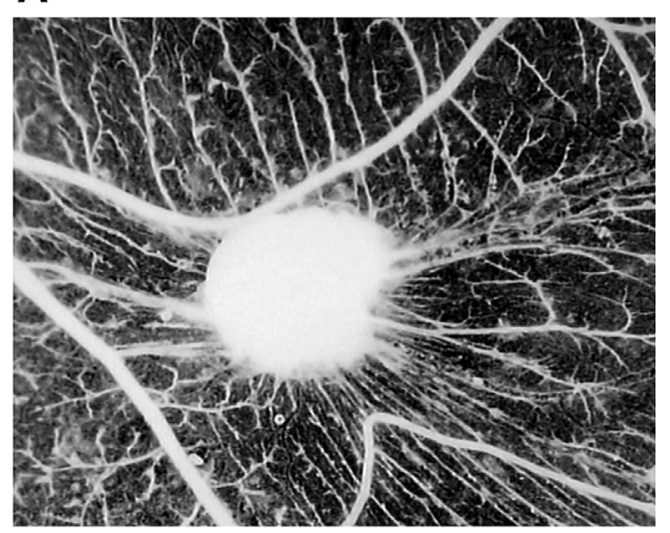

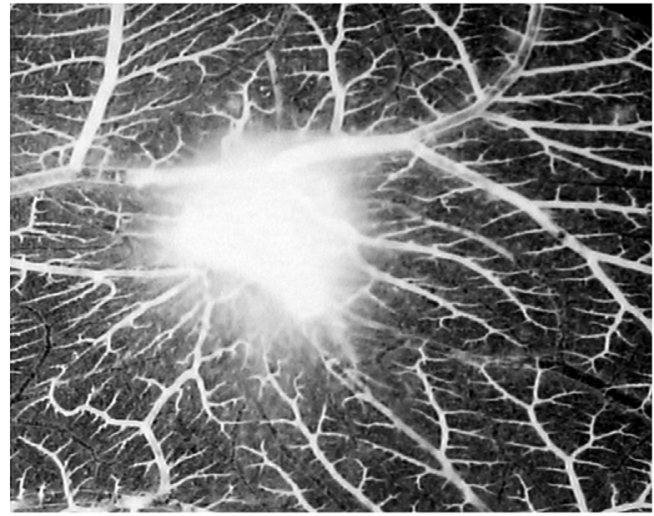

B

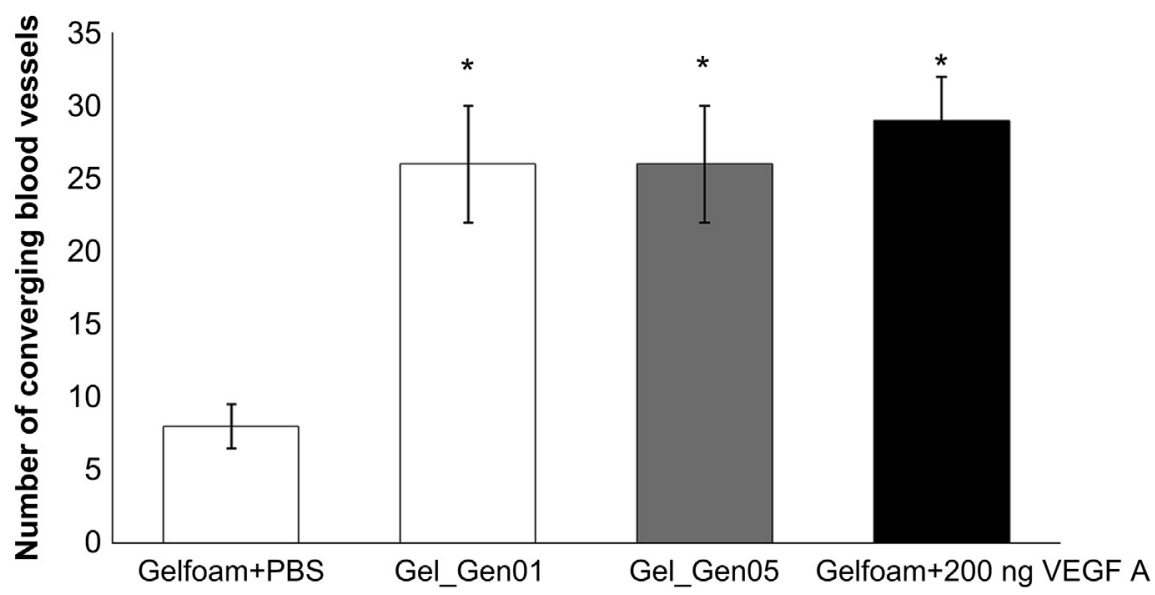

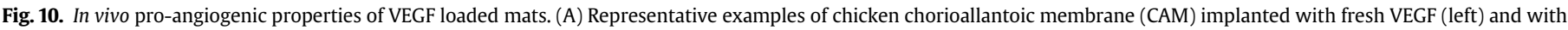

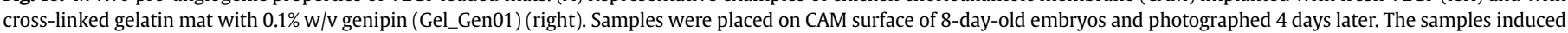

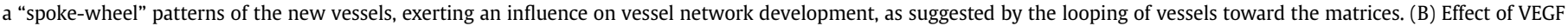
loaded mats on the number of converging blood vessels 4 post-implantation days. ${ }^{*} p<0.05$ versus negative control.

balanced salt solution usually used for hMSCs cultures. Interestingly, Gel_Gen01 mat showed a temporal stability in HBSS throughout the whole testing period that was not observed for the swelling measurements, in addition the absence of degradation was also verified by using Milli-Q water (data not shown). This result might be related to $(i)$ the light cross-linking condition, (ii) the ionic strength of the solutions: it was reported that ions may be capable of disrupting gelatin network [28] thus contributing to the observed temporal instability of the mat, (iii) the volumes considered for the two studies ( $20 \mathrm{ml}$ of PBS, $0.2 \mathrm{ml}$ of HBSS), and (iv) the eventual refreshing of the medium that was carried out for the VEGF release measurements, but not for the swelling test. These conditions, even if a detailed analysis is needed and is in progress, indicate the role of the environment on the functional response of the investigated samples, and might contribute to define a suitable

Table 2

Analysis of the expression of specific antigens, made by flow cytometric evaluation, of hMSCs incubated with loaded or released VEGF for 7 days.

\begin{tabular}{llll}
\hline & CD 44 (\%) & CD90 (\%) & HLA-I (\%) \\
\hline Negative control & $99.38 \pm 0.63$ & $98.30 \pm 1.41$ & $92.83 \pm 6.28$ \\
Positive control & $99.33 \pm 0.70$ & $98.38 \pm 1.89$ & $91.43 \pm 6.86$ \\
Gel_Gen01 & $99.43 \pm 0.42$ & $99.17 \pm 0.76$ & $95.23 \pm 2.41$ \\
Gel_Gen05 & $99.37 \pm 0.65$ & $95.33 \pm 5.76$ & $94.93 \pm 2.60$ \\
7 days & $99.65 \pm 0.57$ & $98.40 \pm 1.20$ & $97.08 \pm 2.58$ \\
14 days & $99.43 \pm 0.57$ & $98.25 \pm 0.97$ & $95.20 \pm 6.04$ \\
\hline
\end{tabular}

in vitro experimental protocol. In this regard, a dissolution study was performed by Moffat and Marra [40] considering poly(ethylene glycol) hydrogels cross-linked with genipin and showing that the dissolution rate was dependent by concentration, mass, and temperature. In particular, the hydrogel samples, cross-linked with $17.6 \mathrm{mM}$ genipin solution, dissolved in 4 days under static condition at $37{ }^{\circ} \mathrm{C}$ and may be referred to the uncross-linked chains rapidly dissolving into the aqueous environment.

Vascular endothelial or progenitor endothelial cells (predifferentiated stem cells that have the potential to proliferate and differentiate into mature endothelial cells) are usually used to evaluate the efficacy of new strategies for revascularization [41]. However, their use in therapy is hampered by their limited expansion capacity and lack of autologous sources. Attracting and localizing mesenchymal stromal cells, which have been demonstrated to be promising source for obtaining endothelial cells able to create stable vascular networks [42,43], could be a novel strategy for endothelialization. For this reason, the potential of genipin cross-linked gelatin mats to act as a bioresorbable platform for the long-term release of VEGF on cultures of human bone marrow MSCs was here investigated.

hMSCs exposure to VEGF-loaded mats showed an increased cellular viability, which resulted to be superior (even if not in a significant way) to that induced by fresh VEGF. For evaluating the in vitro angiogenic potential we tested the VEGF loaded mats using migration (to evaluate chemoattractive potential) and Matrigel assays. The 


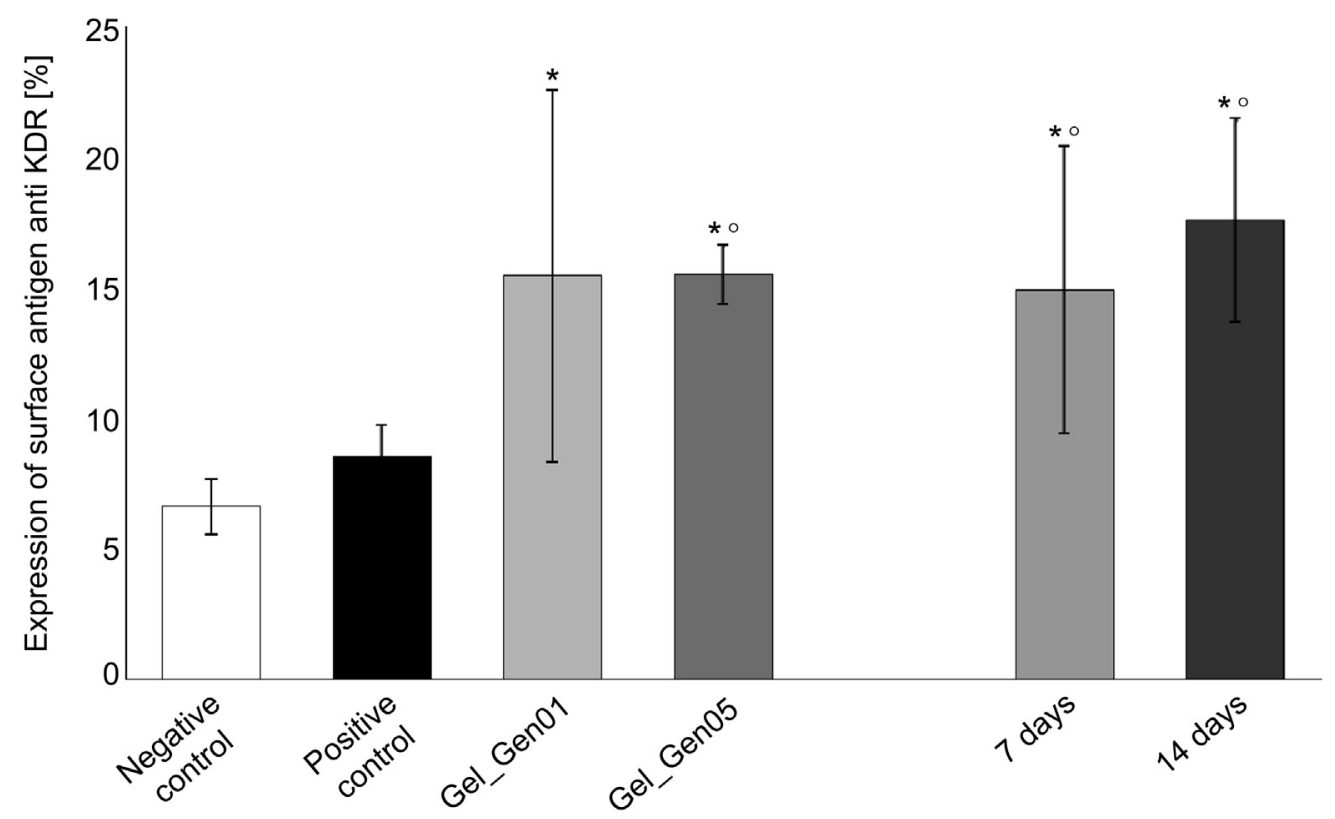

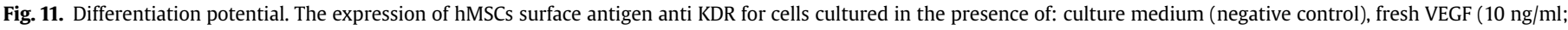

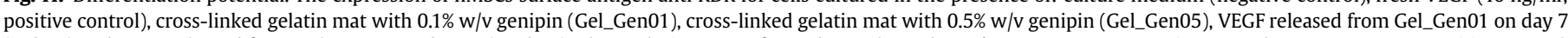

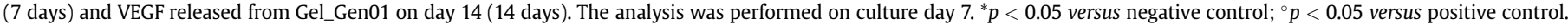

results obtained with VEGF loaded mats were similar to the results observed by exposing hMSCs to fresh VEGF. In particular, for both type of assays, the largest increases were evaluated upon exposure of cells to the VEGF loaded mats and, especially for Gel_Gen01, the increase was significantly $(p<0.05)$ higher, not only with respect to negative control, but also compared to fresh VEGF (positive control).

The present study has also confirmed that VEGF retains bioactive and pro-angiogenic potential for up to 14 days. The VEGF released from cross-linked gelatin mats induced an increase in hMSC viability and capillary network formation on Matrigel with respect to both negative and positive controls.

Chen et al. [44] demonstrated that an optimal temporal evolution of VEGF dose is required in order to promote functional angiogenesis, and the Matrigel assay provides only limited information concerning the dynamics of network formation and maturation. As a consequence, the angiogenic efficiency was proved also in vivo in CAM model. VEGF loaded mats showed a superior angiogenic activity, similar to fresh VEGF, suggesting that during the 4 day experiment, cross-linked gelatin mats were able to release active VEGF.

Considering that VEGF is one of the factors which regulate the differentiation of endothelial cells, it has been evaluated if released VEGF could have any effect on the differentiation of hMSCs: the collected results indicated that a 7 days incubation of hMSCs with VEGF loaded mats or with released VEGF, stimulated an initial differentiation of cells towards endothelial cells. One of the novelty of the current study is the demonstration that hMSCs respond to absorbed VEGF not only by enhanced viability, migration and tube formation, but also by differentiating towards endothelial pathway. As such, this approach could be a useful platform to boost hMSCs towards vascularization. Another interesting outcome of this study was that there was no significant difference in angiogenesis between mats cross-linked with $0.1 \%$ and $0.5 \%$ of genipin. Increasing cross-linking concentration should reduce the swelling ratio with consequent improved retention of VEGF to the mat; consequently, an inverse genipin-dose dependent angiogenic effect can be expected. However, such phenomenon was not observed in our study. It could then be possible that the constant release of $60 \%$ of the adsorbed VEGF up to 7 days (longer time-point for cellular experiments) results sufficient to induce angiogenic response, with consequent similar effects for Gel_Gen01 and Gel_Gen05. It is possible to speculate that the mats with high cross-linking concentration will maintain the optimal release rate of VEGF for extended duration at time-points beyond than 28 days (the longer time-point evaluated for the VEGF release). These findings suggest that optimizing the concentration of the cross-linking agent could be an important aspect to enhance long-term angiogenic response.

\section{Conclusions}

The adsorption of VEGF on genipin cross-linked gelatin mats boosted and induced early angiogenesis. The here reported findings, even if further studies involving animal models will be necessary to draw final conclusions, could contribute to optimize VEGF delivery for enhancing angiogenesis. This can be also regarded as a suitable approach to plan an effective therapeutic strategy to improve the clinical outcome of implanted tissue engineered scaffolds.

\section{Acknowledgments}

This work was supported by European Project FP7-NMP-2011SMALL-5: BIOtrachea, Biomaterials for Tracheal Replacement in Age-related Cancer via a Humanly Engineered Airway, (No. 280584-2) to PM and by European Union Seventh Framework Programme (FPT7/2007-2013) under grant agreement no 278570 to DR.

\section{References}

[1] Baiguera S, Ribatti D. Endothelialization approaches for viable engineered tissues. Angiogenesis 2013;16:1-14.

[2] O'Brien FJ, Harley BA, Yannas IV, Gibson LJ. The effect of pore size on cell adhesion in collagen-GAG scaffolds. Biomaterials 2005;26:433-41.

[3] Rickert D, Moses MA, Lendlein A, Kelch S, Franke RP. The importance of angiogenesis in the interaction between polymeric biomaterials and surrounding tissue. Clin Hemorheol Microcirc 2003;28:175-81.

[4] Pieper JS, Hafmans T, van Wachem PB, van Luyn MJ, Brouwer LA, Veerkamp JH, et al. Loading of collagen-heparan sulfate matrices with bFGF 
promotes angiogenesis and tissue generation in rats. $\mathrm{J}$ Biomed Mater Res 2002;62:185-94.

[5] Jungebluth P, Moll G, Baiguera S, Macchiarini P. Tissue-engineered airway: a regenerative solution. Clin Pharmacol Ther 2012;91:81-93.

[6] Jungebluth P, Bader A, Baiguera S, Möller S, Jaus M, Lim ML, et al. The concept of in vivo airway tissue engineering. Biomaterials 2012;33:4319-26.

[7] Zhang YZ, Venugopal J, Huang ZM, Lim CT, Ramakrishna S. Crosslinking of the electrospun gelatin nanofibers. Polymer 2006;47:2911-7.

[8] Tanaka A, Nagate T, Matsuda H. Acceleration wound healing by gelatin film dressings epidermal growth factor. J Vet Med Sci 2005;67:909-13.

[9] Chen FM, Zhao YM, Wu H, Deng ZH, Wang QT, Zhou W, et al. Enhancement Periodontal Tissue regeneration by locally controlled delivery of insulin-like growth factor-I from dextran-co-gelatin microspheres. J Control Release 2006;114:209-22.

[10] Igai H, Chang SS, Gotoh M, Yamamoto Y, Yamamoto M, Tabata Y, et al. Widespread and early tracheal cartilage regeneration by synchronous slow release of b-FGF and BMP-2. ASAIO J 2009;55:266-70.

[11] Sung HW, Huang RN, Huang LL, Tsai CC. In vitro evaluation of cytotoxicity of a naturally occurring cross-linking reagent for biological tissue fixation. J Biomater Sci Polym Ed 1999;10:63-78.

[12] Sung HW, Chang WH, Ma CY, Lee MH. Crosslinking of biological tissues using genipin and/or carbodiimide. J Biomed Mater Res A 2003;64:427-38.

[13] Zhang K, Qian Y, Wang H, Fan L, Huang C, Yin A, et al. Genipin-crosslinked silk fibroin/hydroxybutyl chitosan nanofibrous scaffolds for tissue-engineering application. J Biomed Mater Res A 2010;95:870-81.

[14] Haag J, Baiguera S, Jungebluth P, Barale D, Del Gaudio C, Castiglione F, et al Biomechanical and angiogenic properties of tissue-engineered rat trachea using genipin cross-linked decellularized tissue. Biomaterials 2012;33:780-9.

[15] Huang M, Vitharana SN, Peek LJ, Coop T, Berkland C. Polyelectrolyte complexes stabilize and controllably release vascular endothelial growth factor. Biomacromolecules 2007;8:1607-14.

[16] Chung YI, Kim SK, Lee YK, Park SJ, Cho KO, Yuk SH, et al. Efficient revascularization by VEGF administration via heparin-functionalized nanoparticlefibrin complex. J Control Release 2010;143:282-9.

[17] Del Gaudio C, Bianco A, Folin M, Baiguera S, Grigioni M. Structural characterization and cell response evaluation of electrospun PCL membranes: micrometric versus submicrometric fibers. J Biomed Mater Res A 2009;89:1028-39.

[18] Baiguera S, Del Gaudio C, Jaus MO, Polizzi L, Gonfiotti A, Comin CE, et al. Longterm changes to in vitro preserved bioengineered human trachea and their implications for decellularized tissues. Biomaterials 2012;33:3662-72.

[19] Le Blanc K, Samuelsson H, Lönnies L, Sundin M, Ringdén O. Generation of immunosuppressive mesenchymal stem cells in allogeneic human serum. Transplantation 2007;84:1055-9.

[20] Urbani S, Caporale R, Lombardini L, Bosi A, Saccardi R. Use of CFDA-SE for evaluating the in vitro proliferation pattern of human mesenchymal stem cells. Cytotherapy 2006;8:243-53.

[21] Ribatti D, Nico B, Vacca A, Presta M. The gelatin sponge-chorioallantoic membrane assay. Nat Protoc 2006;1:85-91.

[22] Baiguera S, Macchiarini P, Ribatti D. Chorioallantoic membrane for in vivo investigation of tissue engineered construct biocompatibility. J Biomed Mater Res B Appl Biomater 2012;100:1425-34.

[23] Hashim DM, Che Man YB, Norakasha R, Shuhaimi M, Salmah Y, Syahariza ZA Potential use of Fourier transform infrared spectroscopy for differentiation of bovine and porcine gelatins. Food Chem 2010;118:856-60.

[24] Qian YF, Zhang KH, Chen F, Ke QF, Mo XM. Cross-linking of gelatin and chitosan complex nanofibers for tissue-engineering scaffolds. J Biomater Sci Polym Ed 2011;22:1099-113.

[25] Young S, Wong M, Tabata Y, Mikos AG. Gelatin as a delivery vehicle for the controlled release of bioactive molecules. J Control Release 2005;109:256-74.
[26] Panzavolta S, Gioffrè M, Focarete ML, Gualandi C, Foroni L, Bigi A. Electrospun gelatin nanofibers: optimization of genipin cross-linking to preserve fiber morphology after exposure to water. Acta Biomater 2011;7:1702-9.

[27] Ravichandran R, Seitz V, Reddy Venugopal J, Sridhar R, Sundarrajan S, Mukherjee $S$, et al. Mimicking native extracellular matrix with phytic Acidcrosslinked protein nanofibers for cardiac tissue engineering. Macromol Biosci 2013;13:366-75.

[28] Zha Z, Teng W, Markle V, Dai Z, Wu X. Fabrication of gelatin nanofibrous scaffolds using ethanol/phosphate buffer saline as a benign solvent. Biopolymers 2012;97:1026-36.

[29] Zhang S, Huang Y, Yang X, Mei F, Ma Q Chen G, et al. Gelatin nanofibrous membrane fabricated by electrospinning of aqueous gelatin solution for guided tissue regeneration. J Biomed Mater Res A 2009;90:671-9.

[30] Skotak M, Noriega S, Larsen G, Subramanian A. Electrospun cross-linked gelatin fibers with controlled diameter: the effect of matrix stiffness on proliferative and biosynthetic activity of chondrocytes cultured in vitro. J Biomed Mater Res A 2010;95:828-36.

[31] Frohbergh ME, Katsman A, Botta GP, Lazarovici P, Schauer CL, Wegst UG, et al. Electrospun hydroxyapatite-containing chitosan nanofibers crosslinked with genipin for bone tissue engineering. Biomaterials 2012;33:9167-78.

[32] Ghajar CM, Blevins KS, Hughes CC, George SC, Putnam AJ. Mesenchymal stem cells enhance angiogenesis in mechanically viable prevascularized tissues via early matrix metalloproteinase upregulation. Tissue Eng 2006;12:2875-88.

[33] Kim HN, Kang DH, Kim MS, Jiao A, Kim DH, Suh KY. Patterning methods for polymers in cell and tissue engineering. Ann Biomed Eng 2012;40:1339-55.

[34] Mason BN, Starchenko A, Williams RM, Bonassar LJ, Reinhart-King CA. Tuning three-dimensional collagen matrix stiffness independently of collagen concentration modulates endothelial cell behavior. Acta Biomater 2013;9: 4635-44.

[35] Singh S, Wu BM, Dunn JC. Delivery of VEGF using collagen-coated polycaprolactone scaffolds stimulates angiogenesis. J Biomed Mater Res A 2012;100:720-7.

[36] Moioli EK, Clark PA, Chen M, Dennis JE, Erickson HP, Gerson SL, et al. Synergistic actions of hematopoietic and mesenchymal stem/progenitor cells in vascularizing bioengineered tissues. PLoS One 2008;3:e3922.

[37] Freeman I, Cohen S. The influence of the sequential delivery of angiogenic factors from affinity-binding alginate scaffolds on vascularization. Biomaterials 2009;30:2122-31.

[38] Wernike E, Montjovent MO, Liu Y, Wismeijer D, Hunziker EB, Siebenrock KA, et al. VEGF incorporated into calcium phosphate ceramics promotes vascularisation and bone formation in vivo. Eur Cell Mater 2010;19:30-40.

[39] Lindhorst D, Tavassol F, von See C, Schumann P, Laschke MW, Harder Y, et al. Effects of VEGF loading on scaffold-confined vascularization. J Biomed Mater Res A 2010;95:783-92.

[40] Moffat KL, Marra KG. Biodegradable poly(ethylene glycol) hydrogels crosslinked with genipin for tissue engineering applications. J Biomed Mater Res B Appl Biomater 2004;71:181-7.

[41] Grieb G, Groger A, Piatkowski A, Markowicz M, Steffens GC, Pallua N. Tissue substitutes with improved angiogenic capabilities: an in vitro investigation with endothelial cells and endothelial progenitor cells. Cells Tissues Organs 2010;191:96-104.

[42] da Silva Meirelles L, Caplan AI, Nardi NB. In search of the in vivo identity of mesenchymal stem cells. Stem Cells 2008;26:2287-99.

[43] Janeczek Portalska K, Leferink A, Groen N, Fernandes H, Moroni L, van Blitterswijk C, et al. Endothelial differentiation of mesenchymal stromal cells. PLoS One 2012;7:e46842.

[44] Chen RR, Silva EA, Yuen WW, Mooney DJ. Spatio-temporal VEGF and PDGF delivery patterns blood vessel formation and maturation. Pharm Res 2007;24: 258-64. 